\title{
Aktuelles rund um Imatinib
}

\section{Aktuelle Therapiekonzepte und zukünftige Entwicklungen bei gastrointestinalen Stromatumoren (GIST)}

Die Voraussetzungen für eine so genannte «molecular targeted therapy» sind die Expression des Zielmoleküls im Tumor, die onkogene Aktivität des Zielmoleküls und die spezifische Hemmbarkeit dieses onkogen aktiven
Moleküls. Dr. Peter Reichardt, Universitätsklinikum Charité, Berlin, beschrieb während des Satellitensymposiums «Neue Standards in der molecular targeted therapy in Hämatologie und Onkologie» während des 26. Deutschen Krebskongresses im Februar 2004 in Berlin, wie diese Voraussetzungen bei der Therapie von GIST mit Imatinib (Handelsname Glivec) auf ideale Weise gegeben sind.

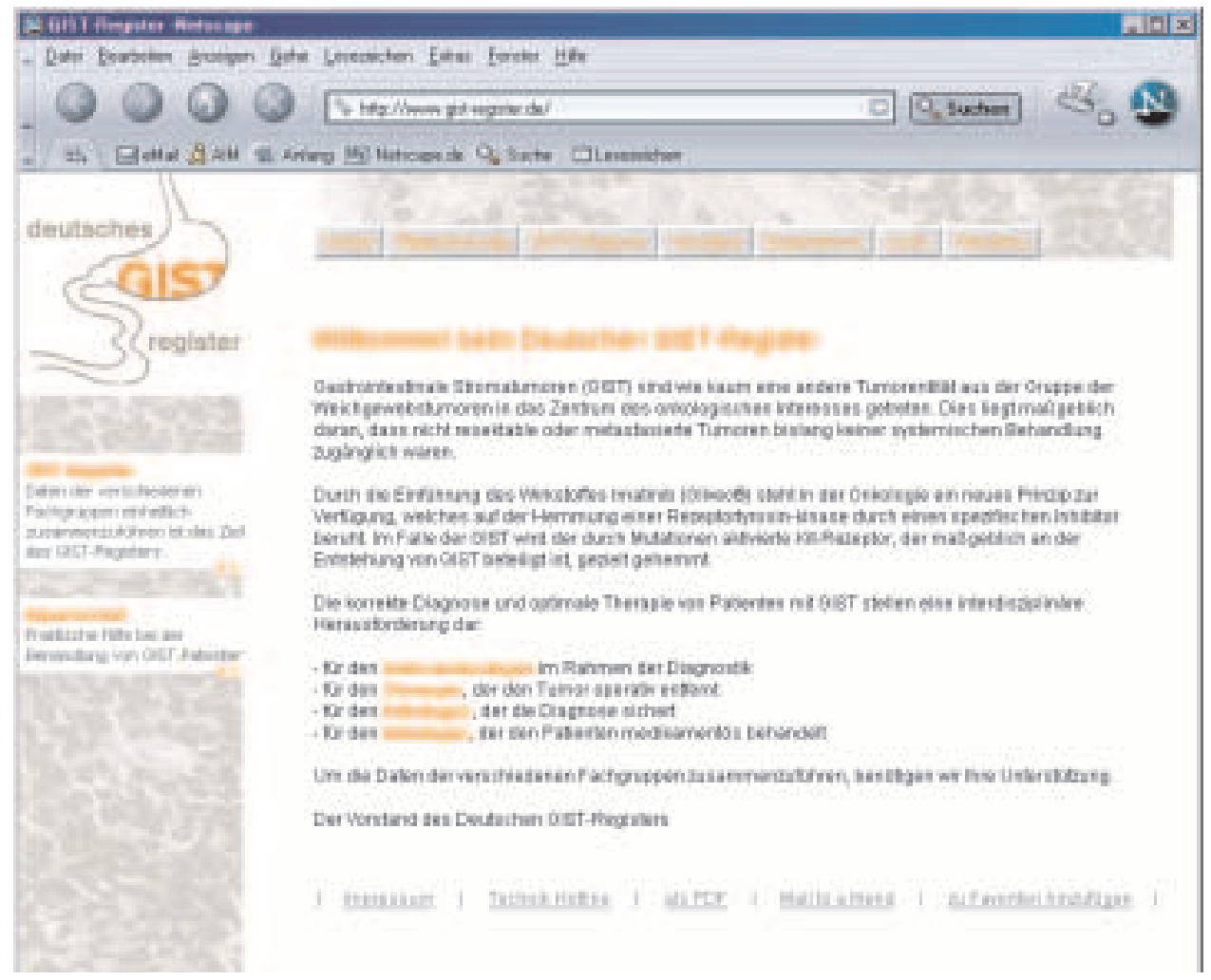

Abb. 1. www.gist-register.de wurde im April 2004 online gestellt. 


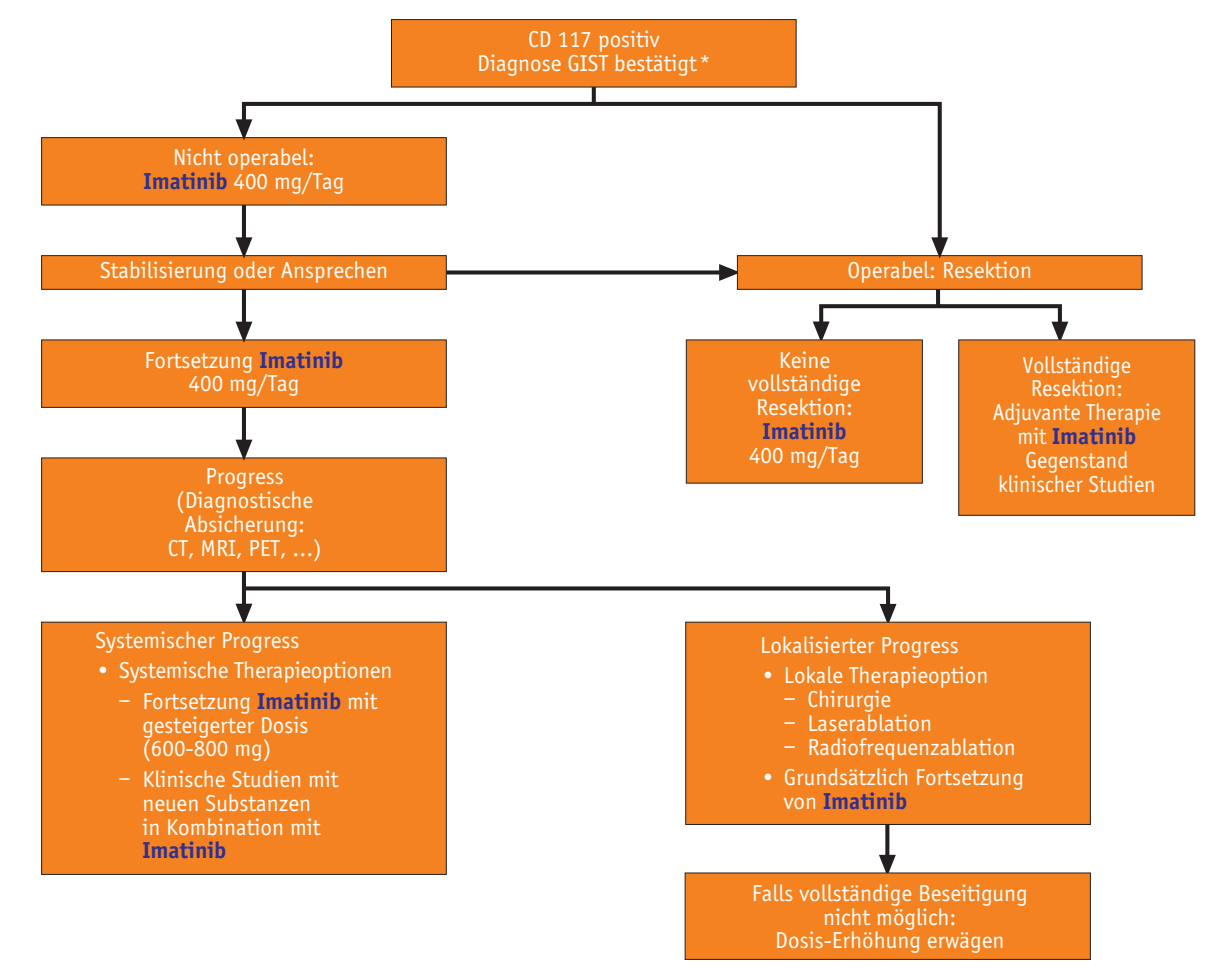

Abb. 2. GIST-Behandlungsalgorithmus: Glivec $^{\circledR}$ auch im Progress nicht absetzen [nach Reichardt, DKK 2004].

*CD117-negative GIST wurden in Einzel-

fällen beschrieben. Behandlung wie GIST.
Das Zielmolekül bei GIST stellt der mutierte transmembranöse Tyosinkinaserezeptor c-kit dar. M. Heinrich vom Health \& Science University Cancer Institute Oregon hat 2003 festgestellt, dass aktivierende Mutationen bei über $90 \%$ der Tumoren nachweisbar sind. Die onkogene Aktivität entsteht bei GIST in etwa $60-70 \%$ der Fälle durch eine Mutation in Exon 11 (weitere Mutationen betreffen Exons 9, 13 und 17), die zu einer ligandenunabhängigen konstitutionellen Daueraktivierung der Tyrosinkinase führt, die wiederum über ein onkogenes Signal den Tumor entstehen lässt. Dieser Prozess wurde im Mausmodell am Memorial SloanKettering Cancer Center in New York nachgestellt, wo durch die sekundäre Induktion von c-kit-Mutationen in einem vorher gesunden Tier GIST hervorgerufen werden konnten. Die Mutation ist also die direkte Ursache der Erkrankung, so Reichardt. Imatinib ist der erste verfügbare selektive Tyrosinkinaseinhibitor, der es ermöglicht, vier Tyrosinkinasen, nämlich ABL, PDGFR-A, ARG und c-kit, zu hemmen. Durch Imatinib wird kompetitiv die ATP-Bindungsstelle gehemmt, was innerhalb 12 Stunden die Signaltransduktion und den proliferativen Stimulus stoppt. GIST waren bislang im fortgeschrittenen Stadium nicht behandelbar. Mit Imatinib erreicht man inzwischen messbare Tumorrückbildungen von 50-70\% und eine Wachstumshemmung von bis zu 90\%. Die Symptome bilden sich bei 80-90\% der Patienten innerhalb von Tagen bis Wochen zurück. Das progressionsfreie Überleben beträgt nach $1 \mathrm{Jahr}$ etwa $70 \%$, nach 2 Jahren etwa $50 \%$. Historische Datenvergleiche zeigen bei mit Imatinib bzw. alternativ behandelten Patienten 2-Jahres-Überlebensraten von 80 bzw. $20 \%$.
In der bislang größten Studie, die in Europa und Australien in insgesamt 56 Institutionen und 13 Ländern durchgeführt wurde, konnte bei 946 Patienten ein Vorteil im Sinne eines längeren progressionsfreien Überlebens durch die Verabreichung von 800 gegenüber $400 \mathrm{mg}$ Imatinib nachgewiesen werden. Eine Progression durch Mutation tritt ungefähr 6 Monate später ein. Ob letztendlich dieser Unterschied einen Überlebensvorteil nach sich zieht, ist noch nicht bekannt. Die Nebenwirkungsrate durch Imatinib zeigte sich in dieser Studie aufgrund des selektiven Wirkmechanismus von Imatinib als sehr gering, so Reichardt.

Die spezifischen Mutationen korrelieren mit dem Therapieergebnis. Mutationen im Exon 11 zeigen das längste progressionsfreie Intervall von etwa 2 Jahren, während Mutationen im Exon 9 mit einem progressionsfreien Intervall von knapp 7 Monaten einhergehen. Liegt keine Mutation im c-kit oder PDGF Rezeptor vor, beträgt das progressionsfreie Intervall weniger als 3 Monate.

Bei lokalisierten Tumoren ist die Operation die Therapie der Wahl. Bei großen Tumoren ist auch bei vollständiger Resektion mit einem hohen Rezidivrisiko zu rechnen, was die Bedeutung adjuvanter Studien mit Imatinib in diesem Zusammenhang unterstreicht. Eine primäre Resektion sollte bei lokal fortgeschrittenen Tumoren unterbleiben. Hier ist eine präoperative Behandlung mit Imatinib in Erwägung zu ziehen.

In Entwicklung sind derzeit Substanzen, die zur Sekundärtherapie bei Resistenzentwicklung gegen Imatinib eingesetzt werden können und der Reaktivierung onkogener Signale entgegenwirken. Solche Substanzen sind beispielsweise SU11248, PKC412 und RAD001. Letztere werden zurzeit im Rahmen von Studien in Berlin untersucht. 
Redaktion ONKOLOGIE: GIST ist eine äußerst seltene Erkrankung. Welche Maßnahmen können helfen, hier neue Erkenntnisse zu gewinnen?

P. Reichardt: Zum einen muss hier festgestellt werden, dass GIST ein zwar sehr seltenes Krankheitsbild darstellt, aber nach neueren Daten mit einer Inzidenz von etwa 15 Neuerkrankungen pro 1,000 000 pro Jahr in derselben Größenordnung liegt wie beispielsweise die chronische myeloische Leukämie (CML). Im Rahmen des TherapieMonitor-Projektes [TherapieMonitor $^{\odot}$ GIST wurde durchgeführt von O.I.S. Lenka Kellermann, Freiburg i.Br.] haben wir in einer repräsentativen Stichprobe von therapeutischen Einrichtungen in Deutschland die GIST-Inzidenz im 4. Quartal 2001 und im 1. Quartal 2002 verbunden mit dem Patientenmanagement untersucht. Es wurden nicht nur therapiebestimmende Onkologen angesprochen, sondern auch Gastroenterologen und Chirurgen. Insgesamt entsprachen die demographischen und diagnostischen Parameter in unserer Dokumentation den publizierten internationalen Daten und bestätigten die Repräsentativität. Die Indikation zur Behandlung mit Imatinib erfolgte in der Regel nach der Metastasierung und Infiltration in das anliegende Gewebe. Die Differenzierung nach Therapieintention und Ergebnis der Operation waren die wichtigsten Faktoren für den Therapiealgorithmus (Abb. 2). Durch die Analyse der Diagnostik und der durchführenden Einrichtungen wurde die Notwendigkeit der Verbesserung von Ergebnisevaluation der primären $\mathrm{OP}$ insbesondere außerhalb der onkologischen Zentren nachgewiesen. Interessanterweise konnte festgestellt werden, dass ein Viertel der Patienten in Krankenhäusern ohne onkologischen Schwerpunkt therapiert und somit unter Umständen keiner onkologischen Therapie zugeführt wurden. Ebenso war das Operationsergebnis bei einem Viertel der Patienten unbekannt, was von großer klinischer Bedeutung sein könnte, da die Qualität der Ergebnisevaluation bei einer R0Resektion von entscheidender Bedeutung für den weiteren Therapieverlauf war.

Wie sehen Sie in diesem Zusammenhang das von Ihnen angesprochene GIST-Register?

Um neue Erkenntnisse zu gewinnen und zu sammeln, wurde das Deutsche GIST-Register ins Leben gerufen. Es ist eine unabhängige Plattform und wurde als vollständig internetbasiertes Tumor-Register etabliert. Hier können Ärzte Patienten anonymisiert registrieren und damit helfen, den Wissensstand rund um GIST zu verbessern. Es fördert zudem die nötige enge interdisziplinäre Zusammenarbeit zwischen
Gastroenterologen, Chirurgen, Pathologen und Onkologen. Das Deutsche GIST-Register ist das erste seiner Art und könnte ein erster Schritt zur Entwicklung eines europäischen GIST-Registers sein. Das deutsche GIST-Register und weitere Informationen zur Nutzung finden Sie unter www.gist-register.de (s. auch Abb. 1).

Sie haben in Ihrem Vortrag die adjuvante Therapie mit Imatinib bei großen Tumoren angesprochen. Wohin kann man derzeit GIST-Patienten zur adjuvanten Therapie mit Imatinib überweisen?

Die Rekrutierung zu einer multizentrischen deutschskandinavischen Phase-II-Studie zur adjuvanten Therapie mit Imatinib bei GIST hat gerade erst begonnen. Das primäre Ziel dieser Studie ist es, das rezidivfreie Überleben bei Patienten mit GIST zu bestimmen, die ein hohes $(>50 \%)$ Rezidivrisiko innerhalb der ersten 5 Jahre nach der Diagnose haben und eine adjuvante Therapie mit Imatinibmesylat über entweder 12 oder 36 Monate erhalten. Jeder Patient in dieser Studie wird mit Imatinib $400 \mathrm{mg} / \mathrm{Tag}$ in Tablettenform behandelt. In Deutschland können Patienten an das deutsche Studienleitzentrum in Berlin überwiesen werden (siehe Kontaktadresse).

\section{Es sind mittlerweile einige Fälle von progredientem Krankheitsverlauf unter Imatinibtherapie beschrieben. Welches Procedere ist hier zu empfehlen?}

Die meisten Fälle eines progredienten systemischen Krankheitsverlaufs unter Imatinib sind unter der Standardtherapie mit $400 \mathrm{mg}$ Imatinib oder bei Unterdosierung mit weniger als $400 \mathrm{mg}$ Imatinib täglich aufgetreten. Generell gilt bei progredientem Krankheitsverlaufs, dass in erster Linie eine Dosissteigerung auf 600 bis $800 \mathrm{mg}$ Imatinib erwogen werden sollte. Erste Studiendaten zeigen den Erfolg dieses Konzepts. Sollte diese Dosissteigerung nicht zum ausreichenden Erfolg führen, sind sekundäre Therapiekonzepte wie die Kombination von Imatinib mit PKC412 oder von Imatinib mit RAD001 in Erwägung zu ziehen. Beide genannten Konzepte sind Gegenstand laufender Studien am Zentrum Berlin.

\section{Wir danken Ihnen für dieses Gespräch.}

Studie: Adjuvanter Einsatz von Imatinib bei resezierbaren GIST mit hohem Rezidivrisiko

\section{Studiensekretariat:}

Frau Simone Micheel

Medizinische Klinik m.S.

Hämatologie, Onkologie und Tumorimmunologie

Robert-Rössle-Klinik

Lindenberger Weg 80, D-13125 Berlin 


\section{Imatinib als mögliche Therapie beim} Glioblastom

Dr. G. Dresemann, Franz-Hospital, Dülmen, stellte auf dem Satellitensymposium seine Therapieerfolge mit Imatinib beim Glioblastoma multiforme vor. Das Glioblastoma, so Dresemann, weist in Deutschland eine Inzidenz von 2 Neuerkrankungen pro 100000 Einwohner mit einer auffälligen doppelgipfeligen Altersverteilung in der 3.-4. Dekade und jenseits des 60. Lebensjahrs auf, wobei Männer bevorzugt betroffen sind. Derzeit bevorzugte Therapien sind die mikrochirurgische Therapie, die konsolidierende Bestrahlung und die Chemotherapie. Die Strahlentherapie zeigt unabhängig von ihrer Applikationsart eine unklare Ansprechrate von $30-40 \%$ auf. Die Chemotherapie hat in der Primärtherapie eine Ansprechrate von etwa 30\%, insgesamt beträgt die Überlebensdauer bei einem Glioblastom derzeit ca. 1 Jahr. Die Strahlentherapie und die Chemotherapie führen zu einer mittleren Lebensverlängerung von 3 Monaten.

Als Rationale für den Einsatz von Imatinib nennt Dresemann die Unmöglichkeit einer R0-Resektion aufgrund der anatomischen Verhältnisse, die niedrige Effizienz der Strahlentherapie und der Chemotherapie, die kurze Gesamtüberlebenszeit und die c-kit- bzw. PDGF-R-Positivität des Tumors.

Die histochemischen Charakteristika des Glioblastoms haben letztendlich zum monotherapeutischen Einsatz von Imatinib beim Glioblastom geführt. Dresemann vermutet, das Problem dieser leider nicht erfolgreichen Studien liege in der Barriere durch die Blut-HirnSchranke. Er erklärt, dass die Blut-Hirn-Schranke vermutlich als stoffwechselaktiver Pumpenmechanismus der Endothelzellen der Hirnkapillaren zu verstehen sei, der durch einfache Moleküle wie Hydroxyurea beeinflussbar zu sein scheint. Diese Vermutung war es schließlich, die Dresemann veranlasste, Hydroxyurea in Kombination mit Imatinib als medikamentöse Therapie beim Glioblastom einzusetzen, nicht zuletzt auch aufgrund des bekannt günstigen Nebenwirkungsprofils der Kombination.

Dresemann schildert zahlreiche Einzelfälle, bei denen er Imatinib nach erfolgloser Vortherapie und Ausreizung aller relevanten Therapieoptionen eingesetzt hat. Bei 26 Patienten konnte in 9 Fällen eine Stabilisierung und in 2 Fällen eine partielle Remission erreicht werden. Die mittlere Zeitspanne bis zum Progress betrug 27 Wochen, wobei 4 Patienten bis zu 105 Wochen weiterhin ohne Progress blieben. In einer seiner Einzelfalldarstellungen schildert Dresemann auch die erfolgreiche Dosiserhöhung von Imatinib auf $600 \mathrm{mg}$ bei weiterhin progredientem Verlauf unter Therapie mit $400 \mathrm{mg}$ Imatinib. Zusammenfassend beschreibt er bei mehr als $30 \%$ der Patienten keine oder eine langsame Progredienz im Sinne einer Down-Regulation des Malignitätsgrads. Das progressionsfreie Überleben scheint nicht von der Remissionsqualität abzuhängen. Dresemanns hoffnungsvoll stimmende Daten werden derzeit in einer Studie bei primär stabilen Patienten mit einem adjuvanten Einsatz von $600 \mathrm{mg}$ Imatinib plus 1000 mg Hydroxyurea geprüft.

\section{Hilft Imatinib bei AML oder Transplantation?}

Ebenfalls während des Satellitensymposiums stellte Prof. Dr. G. Ehninger, Carl-Gustav-Carus-Universität Dresden, neue Studiendaten vor, die den Einsatz von Imatinib in der Therapie der akuten myeloischen Leukämie (AML) und bei der allogenen Transplantation rechtfertigen könnten.

Studien zum Einsatz von Imatinib bei AML sind durch die Überexpression von CD117 und die gelegentlich vorkommende Translokation mit Beteiligung von PDGF (platelet-derived growth factor) begründet. Am MD Anderson Cancer Center, Houston, wurden 18 Patienten mit AML $(\mathrm{n}=8)$ oder myelodysplastischem Syndrom MDS (MDS; $\mathrm{n}=10$ ) mit Imatinib $400 \mathrm{mg} /$ Tag behandelt. Ein hämatologisches Ansprechen wurde nach einer medianen Beobachtungszeit von 60 Tagen bei keinem Patienten beobachtet. T. Fischer et al., Mainz, behandelten 19 Patienten mit c-kit(CD117)-positiver AML (>30\% c-kitpositiv) mit Imatinib $600 \mathrm{mg} / \mathrm{Tag} .4$ Patienten sprachen an: 2 komplette Remissionen, 1 partielle Remission und 1 Blastenfreiheit im Knochenmark. Die mediane Remissionsdauer betrug 108 Tage. In weiteren Studien sollte nun in einer größeren Patientengruppe der Stellenwert von Imatinib auch in der Kombinationstherapie bestimmt werden.

In der aktuellen Studie der GMALL wird Imatinib in Kombination mit Chemotherapie bei Philadelphia-positiver $(\mathrm{Ph}+)$ akuter lymphatischer Leukämie (ALL) bis zum Erreichen einer kompletten Remission eingesetzt. Aufgrund der hohen Rezidivrate wird anschließend eine allogene hämatopoetische Stammzelltransplantation angestrebt. Die Verabreichung von Imatinib wird dann bis zum sicheren Nachweis einer molekularen Vollremission fortgesetzt.

Die hervorragende Wirksamkeit von Imatinib bei der chronischen myeloischen Leukämie hat zu einer deutlichen Abnahme der allogenen Transplantation bei dieser Indikation geführt. Eine primäre Transplantationsindikation wird noch bei jüngeren Patienten und eine sekundäre bei Versagen der Imatinib-Behandlung diskutiert.

Wird nach allogener Transplantation unter Verwendung einer empfindlichen PCR noch eine Resterkrankung detektiert oder tritt ein Krankheitsrückfall auf, so wurden bisher «klassisch» Spenderlymphozyten in steigender Dosierung verabreicht. Von mehreren Autoren wurde bei dieser Indikation eine gute Wirksamkeit von Imatinib berichtet. Möglicherweise kann Imatinib die Gabe von Spenderlymphozyten bei der Mehrzahl der Patienten ersetzen und so das Risiko einer Graft-versus-HostErkrankung vermeiden. 


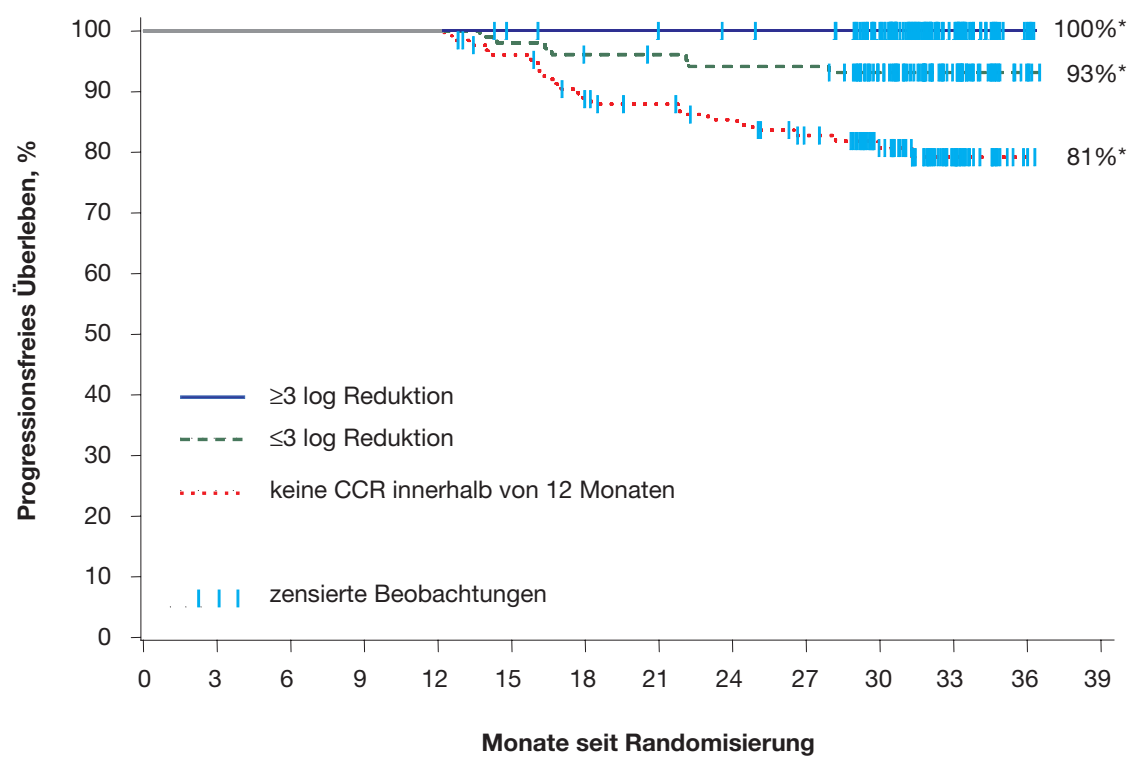

Abb. 3. IRIS Q-PCR Studie: Progressionsfreies Überleben in Abhängigkeit der Bcr-Abl-Reduktion nach 12 Monaten Primärtherapie mit Glivec ${ }^{\circledR}$ [Radich J et al.: Blood 2003;102:181a und Präsentation ASH 2003].

*Wahrscheinlichkeit nach 30 Monaten.

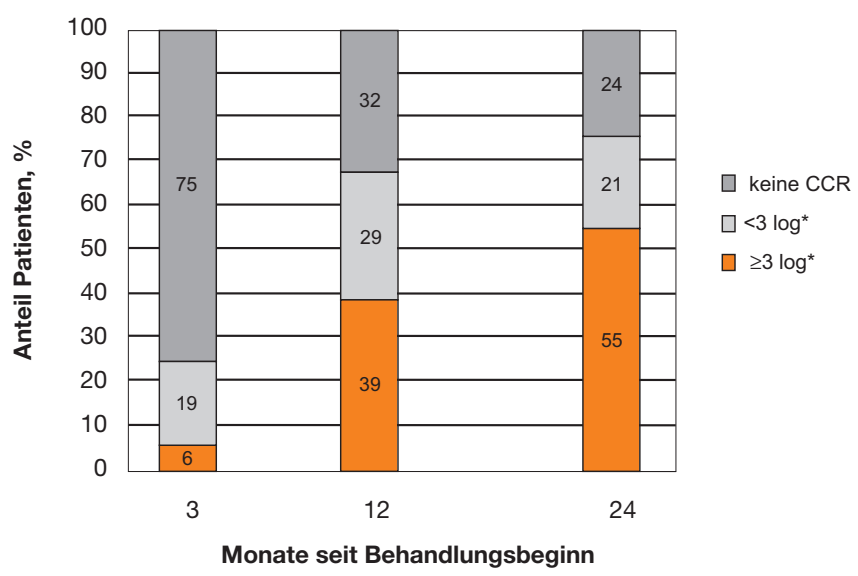

Abb. 4. IRIS Q-PCR Studie: Log-Reduktion Bcr-Abl unter Primärtherapie mit Glivec ${ }^{\circledR}$ [nach Radich J et al.: Blood 2003;102:181a und Präsentation ASH 2003].

*Reduktion Bcr-Abl (inkl. CCR).

\section{Hemmung der Bcr-Abl-Tyrosinkinase bei CML durch Imatinib}

Prof. Dr. A. Hochhaus, Universitätsklinikum Mannheim, diskutierte während des Satellitensymposiums aktuelle Ergebnisse und offene Fragen hinsichtlich der CML-Therapie mit Imatinib. In Deutschland steht Imatinib als Sekundärtherapie im Rahmen von Phase-II-Studien seit fast 5 Jahren, als Primärtherapie seit $2000 \mathrm{im}$ Rahmen der IRIS-Studie zur Verfügung. Die aktuellen Ergebnisse für die Primär- und Sekundärtherapie der CML mit Imatinib wurden auf dem ASH 2003 präsentiert. Die mediane Beobachtungszeit liegt bei 30 bzw. 40 Monaten. In der IRISStudie wurde Imatinib in der Initial-Dosierung von 400 $\mathrm{mg}$ als Primärtherapie bei neu diagnostizierter CML in der chronischen Phase untersucht und mit der Kombina- tion Interferon- $\alpha+$ Ara-C verglichen (insgesamt $1106 \mathrm{~Pa}$ tienten) (Abb. 3, 4). Durch die Primärtherapie mit Imatinib wurde bei $97 \%$ der Patienten eine komplette hämatologische Remission (CHR) erzielt. Noch wichtiger ist das Erreichen einer kompletten zytogenetischen Remission (CCR, $\mathrm{Ph}+0 \%$ ), das mit Imatinib als Primärtherapie bei $82 \%$ der Patienten induziert werden konnte. Die CCRRate hat dabei in der IRIS-Studie über den gesamten Beobachtungszeitraum zugenommen.

Eine Abnahme der Tumorlast um mindestens 3 Zehnerpotenzen ( $\geq 3$ log-Stufen Reduktion bzw. Quotient Bcr$\mathrm{Abl} / \mathrm{Abl}<0,1 \%$ ) gilt als wichtiges Therapieziel und wurde durch die Primärtherapie mit Imatinib nach 24 Monaten bei $55 \%$ der Patienten erreicht. Die prognostische Relevanz dieser molekularen Remission konnte in der IRISStudie nachgewiesen werden. Alle Patienten (100\%) mit einer CCR und einer $\geq 3$ log-Stufen-Reduktion von Bcr$\mathrm{Abl}$ innerhalb von 12 Monaten sind über den gesamten Beobachtungszeitraum progressionsfrei.

Wenn nur die CML-bedingten Todesfälle berücksichtigt werden, liegt das Überleben nach 30 Monaten mit Imatinib als Primärtherapie bei $98 \%$ und ist dem Vergleichsarm Interferon + Ara-C signifikant überlegen $(95 \%, p=$ $0,02)$. Das Gesamtüberleben nach 30 Monaten mit Imatinib als Primärtherapie beträgt $95 \%$.

Eine höhere Initial-Dosierung von 600 und $800 \mathrm{mg}$ Imatinib in der Primärtherapie wurde in zwei Studien untersucht und mit konsekutiven Patientenkohorten mit Imatinib in Standarddosierung (400 mg) verglichen. Eine Erhöhung der Dosierung auf $800 \mathrm{mg}$ bewirkt zu Beginn der Therapie ein stärkeres zytogenetisches Ansprechen. Die Gabe von 800 mg Imatinib ist leicht möglich, da es mittlerweile eine $400 \mathrm{mg}$ Imatinib-Tablette gibt.

Die CML-Studiengruppe untersucht in Deutschland im Rahmen der CML IV-Studie die Kombination von Imatinib mit anderen Substanzen. Es erfolgt eine Randomisierung in Primärtherapie mit Imatinib, Imatinib + Interfe- 
ron, Imatinib + nierig dosiertes Ara-C und in Sekundärtherapie mit Imatinib nach Interferon-Versagen. Bei hohem Risiko entfällt der letzte Arm, und stattdessen erfolgt eine Therapie mit $800 \mathrm{mg}$ Imatinib. Beim Versagen dieser Therapieoptionen wird für geeignete Patienten eine allogene Stammzelltransplantation empfohlen.

Im Verlauf der Therapie kann die Prognose anhand des zytogenetischen Ansprechens beurteilt werden. Im Rahmen der IRIS-Studie wurde gezeigt, dass Patienten ohne zytogenetisches Ansprechen nach 3 Monaten noch im Verlauf der weiteren Behandlung eine gute Chance auf eine CCR haben. Diese Wahrscheinlichkeit liegt höher als die einer Progression. Falls nach 6 Monaten noch kein zytogenetisches Ansprechen vorliegt, haben Patienten ein hohes Risiko einer Progression im Vergleich zum Erreichen einer CCR. Das ist daher ein sinnvoller Zeitpunkt, um eine Entscheidung über den weiteren Ablauf der Behandlung zu fällen.

Ein gutes Ansprechen auf Imatinib kann wie folgt auf drei Stufen definiert werden:

- Hämatologisch: Normalisierung von Blutbild und Milzgröße nach 3 Monaten.

- Zytogenetisch: Jegliche zytogenetische Remission $(\mathrm{Ph}+$ $<95 \%$ ) nach 6 Monaten, größere zytogenetische Remission $(\mathrm{Ph}+<35 \%)$ nach 12 Monaten, CCR $(\mathrm{Ph}+0 \%)$ nach 18 Monaten.

- Molekular: $\geq 3$ log-Reduktion Bcr-Abl (Quotient Bcr$\mathrm{Abl} / \mathrm{Abl}<0,1 \%$ ) nach 18 Monaten.

Nach einem guten Ansprechen auf Imatinib, welches die meisten Patienten erreichen, sollte die Therapie in konstanter Dosierung fortgesetzt werden. Aufgrund der Möglichkeit einer Resistenzentwicklung ist eine Dosisreduktion nicht zu empfehlen. Der Abbruch einer Behandlung mit Imatinib ist nur in sehr speziellen Situationen, wie z.B. einem dringenden Kinderwunsch oder bei Nebenwirkungen, zu erwägen. Nach dem Erreichen einer CCR und einer guten molekularen Remission ist eine Stammzellsammlung empfehlenswert. Allerdings gibt es noch keine Erfahrungen über Transplantationen mit gesammelten Stammzellen im Fall eines Rezidivs.

\section{Quelle}

Assoziiertes Symposium «Neue Standards in der molecular targeted therapy in Hämatologie und Onkologie» am 29.02.2004 während des 26. Deutschen Krebskongresses in Berlin.
Impressum

\section{Aktuelles rund um Imatinib}

\section{Beilage zu ONKOLOGIE 27 | 3 | 04}

Mit freundlicher Unterstützung durch Novartis Pharma $\mathrm{GmbH}$.

Die Wiedergabe von Gebrauchsnamen, Handelsnamen, Warenbezeichnungen usw. in dieser Zeitschrift berechtigt auch ohne besondere Kennzeichnung nicht zur Annahme, dass solche Namen im Sinne der Warenzeichen- und Markenschutz-Gesetzgebung als frei zu betrachten wären und daher von jedermann benutzt werden dürfen. Für Angaben von Dosierungsanweisungen und Applikationsformen kann vom Verlag keine Haftung übernommen werden. Derartige Angaben müssen vom jeweiligen Anwender im Einzelfall anhand anderer Literaturstellen auf ihre Richtigkeit überprüft werden.

Alle Rechte vorbehalten.

Ohne schriftliche Genehmigung des Verlags dürfen diese Publikation oder Teile daraus nicht in andere Sprachen übersetzt oder in irgendeiner Form mit mechanischen oder elektronischen Mitteln (einschließlich Fotokopie, Tonaufnahme oder Mikrokopie) reproduziert oder auf einem Datenträger oder Computersystem gespeichert werden.

(C) 2004 by S. Karger Verlag für Medizin und Naturwissenschaften GmbH, Postfach, D-79095 Freiburg und S. Karger AG; Postfach, CH4009 Basel.

Druck: Konkordia GmbH · Das Medienunternehmen 